\title{
System Identification in the Presence of Outliers and Random Noises: a Compressed Sensing Approach
}

\author{
Weiyu Xu ${ }^{\text {a }}$ Er-Wei Bai ${ }^{\text {a,b }}$ Myung Cho ${ }^{\text {a }}$ \\ ${ }^{a}$ Dept. of Electrical and Computer Engineering, University of Iowa, Iowa City, Iowa 52242 \\ ${ }^{\mathrm{b}}$ School of Electronics, Electrical Engineering and Computer Science, Queen's University, Belfast, UK
}

\begin{abstract}
In this paper, we consider robust system identification of FIR systems when both sparse outliers and random noises are present. We reduce this problem of system identification to a sparse error correcting problem using a Toeplitz structured realnumbered coding matrix and prove the performance guarantee. Thresholds on the percentage of correctable errors for Toeplitz structured matrices are established. When both outliers and observation noise are present, we have shown that the estimation error goes to 0 asymptotically as long as the probability density function for observation noise is not "vanishing" around 0 . No probabilistic assumptions are imposed on the outliers.
\end{abstract}

Key words: system identification, least absolute deviation, $\ell_{1}$ minimization, Toeplitz matrix, compressed sensing

\section{Introduction}

In a linear system identification setting, an unknown system parameter vector $\mathbf{x} \in R^{m}$ is often observed through a Toeplitz matrix $H \in R^{n \times m}(n \geq m)$, namely

$$
\mathbf{y}=H \mathbf{x}=\left[\begin{array}{cccc}
u_{1} & u_{0} & \ldots & u_{-m+2} \\
u_{2} & u_{1} & \ldots & u_{-m+3} \\
\vdots & \vdots & \ddots & \vdots \\
u_{n} & u_{n-1} & \ldots & u_{-m+n+1}
\end{array}\right] \mathbf{x}
$$

where $H$ is a Toeplitz matrix with $u_{i},-m+2 \leq$ $i \leq n$, being the system input sequence and $\mathbf{y}=$ $\left(y_{1}, y_{2}, \ldots, y_{n}\right)^{T}$ the system output. In this paper, we denote this system input sequence by a row vector $h=\left(u_{-m+2}, u_{-m+1}, \ldots, u_{n}\right)$.

In this paper, we consider system identification under finite impulse response (FIR) models. Though applicable to control applications where FIR models are used, it is not as complete as IIR models from a control point

\footnotetext{
* This paper was not presented in any IFAC meeting. This work was supported in part by NSF CNS-1329657 and and DoE DE-FG52-09NA29364. The corresponding author is ErWei Bai, Tel:+3193355949 and Fax: +3193356028 .
}

of view. However, identification in the presence of outliers and random noises is not limited to control applications and in fact routinely applied to many other areas, e.g., signal processing and communication [15,22]. Note in those areas, the systems are dominantly and overwhelmingly FIR models and thus the results derived in this paper can be readily applied.

If there is no interference or noise in the observation $\mathbf{y}$, one can then simply recover $\mathbf{x}$ from matrix inversion. However, in applications, the observations $\mathbf{y}$ are corrupted by noises and a few elements can be exposed to large-magnitude gross errors or outliers. Mathematically, when both additive observation noise and outliers are present, the observation $\mathbf{y}$ can be written as

$$
\mathbf{y}=H \mathbf{x}+\mathrm{e}+\mathbf{w}
$$

where e is a sparse outlier vector with $k \ll n$ non-zero elements, and $\mathbf{w}$ is a measurement noise vector with each element usually being assumed to be i.i.d. random variables. We further assume $m$ is fixed and $n$ can increase, which is often the case in system identifications [5].

If only random measurement errors are present, the least-square solutions generally provide an asymptotically good estimate. However, the least-square estimate breaks down in the presence of outliers. Thus, it is necessary to protect the estimates from both random

Preprint submitted to $X X X$ 
noise and outliers. Research along this direction has attracted a significant amount of attention, for example, $[1,4,5,7]$. An effective way is to visually inspect the residual plot and change the obviously erroneous measurements "by hand" to an appropriately interpolated values [5]. The approach does not however always work. The need for human intervention, which prevents automatic/adaptive implementation, is an additional shortcoming of the visual inspection of the residual plot as a means to account for the presence of outliers. Another approach to deal with the outliers was the idea of few violated constraints [1] in the setting of the bounded error parameter estimation. The other two popular methods in the statistical literature to deal with the outliers are the least median squares and the least trimmed squares $[4,12]$. Instead of minimizing the sum of the residual squares, the least median squares yields the smallest value for the median of squared residuals computed from the entire data set and the least trimmed squares tries to minimize the sum of squared residues over a subset of the given data. Both have shown robustness against the outliers $[4,12]$. The problem is their computational complexity. Both algorithms are nonlinear and in fact combinatory in nature. This limits their practical applications if $n$ and/or $m$ are not small or even modest. The most popular way to deal with the outliers in the statistical literature is the the least absolute deviation estimate $\left(\ell_{1}\right.$ minimization $)$ which has been extensively studied $[2,8,9,19]$. Instead of searching for all the $\left(\begin{array}{l}n \\ k\end{array}\right)$ possibilities for the locations of outliers, $[2,8,9]$ proposed to minimize the least absolute deviation,

$$
\hat{\mathbf{x}}=\arg \min _{\xi}\|\mathbf{y}-H \xi\|_{1},
$$

where $\hat{\mathbf{x}}$ is an estimate of $\mathbf{x}$. Under the assumption that the error $\mathrm{e}+\mathbf{w}$ is an i.i.d. random sequence with a common density which has median zero and is continuous and positive in the neighborhood of zero, the difference between the unknown $\mathbf{x}$ and its estimate is asymptotically Gaussian of zero mean [2]. The problem is that the assumption of i.i.d. of median zero on the unknown outliers is very restrictive and seldomly satisfied in reality. We study the least absolute deviation estimator or $\ell_{1}$ minimization from the compressed sensing point of view and show that i.i.d. of median zero on the outliers are unnecessary. In fact only the number of outliers relative to the total number of data length plays a role.

Recovering signals from outliers or errors have been studied $[8,9,18,19]$. In their setting, each element of the $(n-m) \times n$ matrix $A$ such that $A H=0$, is assumed to be i.i.d. random variables following a certain distribution, for example, Gaussian distribution or Bernoulli distribution. These types of matrices have been shown to obey certain conditions such as restricted isometry conditions [8] so that (1.3) can correctly recover $\mathbf{x}$ when there are only outliers present; and can recover $\mathbf{x}$ approximately when both outliers and measurement noise exist. However, in the system identification problem, $H$ has a natural Toeplitz structure and the elements of $H$ are not independent but correlated. The natural question is whether (1.3) also provides performance guarantee for recovering $\mathbf{x}$ with a Toeplitz matrix. We provide a positive answer in this paper. The main contribution of this paper is the establishment of the performance guarantee of Toeplitz structured matrices in parameter estimation in the presence of both outliers and random noises.

With the development of compressed sensing theory in recent years, the role of $\ell_{1}$ regularization has been studied in system identification $[14,15,20-22]$. In these works, system parameters are often assumed to be sparse, and then $\ell_{1}$ regularization can be used to reduce the number of needed samples for system identification. This paper instead considers system identification under sparse outliers without requiring the system state to be sparse. We would like to point out that there is a well known duality between compressed sensing [10,11] and sparse error detection $[8,9]$ : the null space of sensing matrices in compressed sensing corresponds to the tall matrix $H$ in sparse error corrections. Toeplitz and circulant matrices have been studied in compressed sensing in several papers $[14,15]$. In these papers, it has been shown that Toeplitz matrices are good for recovering sparse vectors from undersampled measurements. In contrast, in our model, the signal itself is not sparse and the linear system involved is overdetermined rather underdetermined. Also, the null space of a Toeplitz matrix does not necessarily correspond to another Toeplitz matrix; so the problem studied in this paper is essentially different from those studied in $[14,15]$.

The rest of this paper is organized as follows. In Section 2 , we derive the convergence results when both outliers and random noises are present. In Section 3, we derive the worst-case performance bounds on the number of outliers in $\ell_{1}$ minimization when only outliers are present. In Section 4, we extend our results to nonGaussian inputs in system identification. In Section 5, we provide numerical results and Section 6 concludes the paper by discussing extensions and future directions.

\section{Average-Case Performance Bounds: With Both Outliers and Observation Noises}

We consider the case when both outliers and random observation errors are present and show that, under mild conditions, the identification error $\|\hat{\mathbf{x}}-\mathbf{x}\|_{2}$ goes to 0 , where $\hat{\mathbf{x}}$ is the solution to (1.3).

Theorem 2.1 Let $m$ be a fixed positive integer and $H$ be an $n \times m$ Toeplitz matrix $(m<n)$ in (1.1) with each element $u_{i},-m+2 \leq i \leq n$, being i.i.d. $N(0,1)$ Gaussian 
random variables. Suppose

$$
\mathbf{y}=H \mathbf{x}+\mathrm{e}+\mathbf{w}
$$

where e $i s$ a sparse vector with $k \leq \beta n$ non-zero elements $(\beta<1$ is a constant) and $\mathbf{w}$ is the observation noise vector. For any constant $t>0$, we assume that, with probability 1 as $n \rightarrow \infty$, at least $\alpha(t) n$ (where $\alpha(t)>0$ is a constant depending on $t$ ) elements in $\mathbf{w}+\mathrm{e}$ are no bigger than $t$ in amplitude.

Then $\|\hat{\mathbf{x}}-\mathbf{x}\|_{2} \rightarrow 0$ in probability as $n \rightarrow \infty$, where $\hat{\mathbf{x}}$ is the solution to (1.3).

We remark that, in Theorem 2.1, the condition on the unknown outlier vector is merely $\beta<1$, and the condition on the random noise $\mathbf{w}$ is weaker than the usual condition of having i.i.d. elements with median 0 [2]. In fact, if $\mathbf{w}$ is independent from e, and the elements of $\mathbf{w}$ are i.i.d. random variables following a distribution which is not "vanishing" in an arbitrarily small region around 0 (namely the cumulative distribution function $F(t)$ satisfies that $F(t)-F(-t)>0$ for any $t>0)$. Note that the probability density function $f(t)$ is allowed to be 0 , however), the conditions in Theorem 2.1 will be satisfied. To see that, first observe that $(1-\beta) n$ elements of the outlier vector are zero. If elements of $\mathbf{w}$ are i.i.d. following a probability density function $f(s)$ that is not "vanishing" around $s=0$, with probability converging to one as $n \rightarrow \infty$, at least $[F(t)-F(-t)](1-\beta)(1-\epsilon) n=\alpha(t) n$ elements of the vector $e+\mathbf{w}$ are no bigger than $t$, where $\epsilon>0$ is an arbitrarily small number. Gaussian distributions, exponential distributions, Gamma distributions and many other distributions all satisfy such conditions in Theorem 2.1. This greatly broadens the existing results, e.g., in [2], which requires $f(0)>0$ and does not accommodate outliers. Compared with analysis in compressed sensing [8], this result is for Toeplitz matrix in system identification and applies to observation noises with non-Gaussian distributions. The results in this paper also improve on the performance bounds in [19], by showing that the identification error actually goes to 0 .

Proof (of Theorem 2.1) $\|\mathbf{y}-H \hat{\mathbf{x}}\|_{1}$ can be written as $\|H(\mathbf{x}-\hat{\mathbf{x}})+\mathrm{e}+\mathbf{w}\|_{1}$. We set out to prove that for any constant $t>0$, with probability 1 as $n \rightarrow \infty$, for all $\hat{\mathbf{x}}$ such that $\|\mathbf{x}-\hat{\mathbf{x}}\|_{2}=t,\|H(\mathbf{x}-\hat{\mathbf{x}})+\mathrm{e}+\mathbf{w}\|_{1}>$ $\|\mathrm{e}+\mathbf{w}\|_{1}$, contradicting the assumption that $\hat{\mathbf{x}}$ is the solution to (1.3). This will then imply that $\|\hat{\mathbf{x}}-\mathbf{x}\|_{2} \rightarrow 0$ in probability as $n \rightarrow \infty$.

For every $\hat{\mathbf{x}}$ such that $\|\mathbf{x}-\hat{\mathbf{x}}\|_{2}=t$, we first represent $\mathbf{x}-\hat{\mathbf{x}}=t z$, where $z \in Z=\left\{z \mid\|z\|_{2}=1\right\}$. So we need to check $\|H t z+\mathrm{e}+\mathbf{w}\|_{1}>\|\mathrm{e}+\mathbf{w}\|_{1}$ holds for every $z \in\left\{z \mid\|z\|_{2}=1\right\}$. The difficulties of checking this condition for every $\hat{\mathbf{x}}$ are that the elements of $H$ are not independent random variables, and that this condition must hold for every vector $z \in Z$. To this end, we adopt the following strategy of discretizing the subspace generated by $H$,see $[13,16]$.

We pick a finite set $V=\left\{v_{1}, \ldots, v_{N}\right\}$ called $\gamma$-net on $\left\{z \mid\|z\|_{2}=1\right\}$ for a constant $\gamma>0$ : in a $\gamma$-net, for every point $z$ from $\left\{z \mid\|z\|_{2}=1\right\}$, there is a $v_{l} \in V$ such that $\left\|z-v_{l}\right\|_{2} \leq \gamma$. In fact, for any given $\gamma>0$, there exists a $\gamma$-net $\bar{V}=\left\{v_{1}, \ldots, v_{N}\right\}$ of cardinality less than $\left(1+\frac{2}{\gamma}\right)^{m}[16]$. We first argue that for every discrete point $v_{j}$ from the $\gamma$-net, $\left\|H t v_{j}+\mathrm{e}+\mathbf{w}\right\|_{1}>\|\mathrm{e}+\mathbf{w}\|_{1}$; and then extend the result to $\|\mathbf{x}-\hat{\mathbf{x}}\|_{2} \in t Z$. Define

$g(h, t)=\left\|H t v_{j}+\mathrm{e}+\mathbf{w}\right\|_{1}-\|\mathrm{e}+\mathbf{w}\|_{1}=\sum_{i=1}^{n}\left(\left|l_{i}+t\left(H v_{j}\right)_{i}\right|-\left|l_{i}\right|\right)$,

where $l_{i}=(\mathrm{e}+\mathbf{w})_{i}$ for $1 \leq i \leq n$. We note that $\left(H v_{j}\right)_{i}$ is a Gaussian random variable $N(0,1)$. Let $X$ be a Gaussian random variable $N\left(0, \sigma^{2}\right)$, then for an arbitrary number $l$,

$$
\begin{gathered}
E\{|l+t X|-|l|\}=\frac{2}{\sqrt{2 \pi} t \sigma} \int_{0}^{\infty} x e^{-\frac{(|l|+x)^{2}}{2 t^{2} \sigma^{2}}} d x \\
=\sqrt{\frac{2}{\pi}} t \sigma e^{-\frac{l^{2}}{2 t^{2} \sigma^{2}}}-2|l|\left(1-\Phi\left(\frac{|l|}{t \sigma}\right)\right),
\end{gathered}
$$

which is a decreasing nonnegative function in $|l|$. To see this, just notice that, by symmetry, $E\{|l+t X|-|l|\}=$ $2 E\{(t X-|l|) \mathbf{1}(t X \geq|l|)\}$, where $\mathbf{1}(\cdot)$ is the indicator function, and $2 E\{(t X-|l|) \mathbf{1}(t X \geq|l|)\}$ is a decreasing function in $|l|$.

From now on, we take $\sigma=1$. When $|l| \leq t$ and $\sigma=1$,

$E\{|l+t X|-|l|\}=\sqrt{\frac{2}{\pi}} t e^{-\frac{1}{2}}-2|l|(1-\Phi(1)) \geq 0.1666 t$.

From this, $E\{g(h, t)\}=\sum_{i=1}^{n}\left(\sqrt{\frac{2}{\pi}} t e^{-\frac{\left|l_{i}\right|^{2}}{2 t^{2}}}-2\left|l_{i}\right|(1-\right.$ $\left.\left.\Phi\left(\frac{\left|l_{i}\right|}{t}\right)\right)\right)$. It is not hard to verify that $|g(a, t)-g(b, t)| \leq$ $\sum_{i=-m+2}^{n} t \sqrt{m}\left|a_{i}-b_{i}\right| \leq t \sqrt{m(n+m-1)}\|a-b\|_{2}$, and $g(h, t)$ has a Lipschitz constant (for $h$ ) no bigger than $t \sqrt{m(n+m-1)}$ (for details, please see proof of Lemma 7.1 in the appendix). Let us take $\epsilon>0$ as a sufficiently small constant. Then by the concentration of measure inequality for Lipschitz function of Gaussian random variables (see $[16,17]$. For the inequality statement, please see the proof of Lemma 7.1 in the appendix), we have

$$
\begin{aligned}
& P(g(h, t) \leq \epsilon n) \\
= & P\left(\frac{g(h, t)-E\{g(h, t)\}}{t \sqrt{m(n-m+1)}} \leq \frac{\epsilon n-E\{g(h, t)\}}{t \sqrt{m(n-m+1)}}\right) \\
\leq & 2 e^{-\frac{\left(\epsilon n-\sum_{i=1}^{n}\left[\sqrt{\frac{2}{\pi}} t e^{-\frac{l_{i}^{2}}{2 t^{2}}-2\left|l_{i}\right|\left(1-\Phi\left(\frac{\left|l_{i}\right|}{t}\right)\right)}\right]\right)^{2}}{2 t^{2}(n-m+1) m}} \triangleq 2 e^{-B} .
\end{aligned}
$$


If there exists a constant $\alpha(t)$ such that, as $n \rightarrow \infty$, at least $\alpha(t) n$ elements have magnitudes smaller than $t$, then the numerator in $B$ behaves as $\Theta\left(n^{2}\right)$ and the corresponding probability $P(g(h, t) \leq \epsilon n)$ behaves as $2 e^{-\Theta(n)}$. This is because when $|l| \leq t, \sqrt{\frac{2}{\pi}} t e^{-\frac{|l|^{2}}{2 t^{2}}}-$ $2|l|\left(1-\Phi\left(\frac{|l|}{t}\right)\right) \geq 0.1666 t$; and also because $\epsilon$ is a sufficiently small constant.

So far, we have shown that, with probability $P \leq e^{-c_{1} n}$, $g(h, t) \leq \epsilon n$ holds for each discrete point $v_{j}$ from the $\gamma$ net $V$, where $c_{1}>0$ is a constant which only depends on $\epsilon$. Since there are at most $\left(1+\frac{2}{\gamma}\right)^{m}$ points from the $\gamma$ net, by a simple union bound, with probability at least $P_{n e t}=1-P\left(1+\frac{2}{\gamma}\right)^{m}, g(h, t)>\epsilon n$ holds for all points from the $\gamma$-net $V$. Since $m$ is fixed, then for any constant $\gamma>0$, with probability $1-e^{-c_{2} n}, g(h, t)>\epsilon n$ holds for all points $v_{j}$ from the $\gamma$-net $V$, where $c_{2}>0$ is a constant.

We are on track to show that, with high probability, $\|H t v+\mathrm{e}+\mathbf{w}\|_{1}-\|\mathrm{e}+\mathbf{w}\|_{1}>0.5 \epsilon n$ holds simultaneously for all points $v \in Z$. To do so, we would need Lemma 2.2.

Lemma 2.2 Let $0<\delta<1$ be a constant. Then there exists a constant $c_{3}>0$ (depending on $m$ and $\beta$ ), such that with probability at least $1-e^{-c_{3} n}$, for every $z \in$ $\left\{z \mid\|z\|_{2}=1\right\}$,

$$
(1-\delta) S \leq\|(H z)\|_{1} \leq(1+\delta) S,
$$

where $S=E\left\{\|H z\|_{1}\right\}=n E\{|X|\}$ and $X$ is a random variable following the Gaussian distribution $N(0,1)$.

For the proof of Lemma 2.2, please refer to the appendix.

Let $v$ be an arbitrary point on the unit sphere $\left\{z \mid\|z\|_{2}=\right.$ $1\}$. By the definition of $\gamma$-net, there exists a $v_{j}$ from a $\gamma$-net of cardinality of no more than $\left(1+\frac{2}{\gamma}\right)^{m}$, such that $\left\|v-v_{j}\right\|_{2} \leq \gamma$. Then with high probability, we have

$$
\begin{aligned}
& \|H t v+\mathrm{e}+\mathbf{w}\|_{1}-\|\mathrm{e}+\mathbf{w}\|_{1} \\
\geq & \left\|H t v_{j}+\mathrm{e}+\mathbf{w}\right\|_{1}-\|\mathrm{e}+\mathbf{w}\|_{1}-\left\|H t\left(v-v_{j}\right)\right\|_{1} \\
\geq & \epsilon n-\gamma(1+\delta) S
\end{aligned}
$$

where the first inequality is from the triangle inequality, and the second inequality is from Lemma 2.2. Since $S$ is of the order of $O(n)$, if we take $\delta$ and $\gamma$ to be small enough constants such that $\epsilon n-\gamma(1+\delta) S>0.5 \epsilon n$, then $\|H t v+\mathrm{e}+\mathbf{w}\|_{1}-\|\mathrm{e}+\mathbf{w}\|_{1}>0.5 \epsilon n$ for every $v \in$ $\left\{z \mid\|z\|_{2}=1\right\}$ with high probability $1-e^{-c_{4} n}$, where $c_{4}>0$ is a constant.

Notice if $\left\|H t_{1} v+\mathrm{e}+\mathbf{w}\right\|_{1}-\|\mathrm{e}+\mathbf{w}\|_{1}>0$ for $t_{1} \geq 0$, then necessarily $\left\|H t_{2} v+\mathrm{e}+\mathbf{w}\right\|_{1}-\|\mathrm{e}+\mathbf{w}\|_{1}>0$ for $t_{2}>t_{1}$. This is because $\|H t v+\mathrm{e}+\mathbf{w}\|_{1}-\|\mathrm{e}+\mathbf{w}\|_{1}$ is a convex function in $t \geq 0$ and $\|H t v+\mathrm{e}+\mathbf{w}\|_{1}-\|\mathrm{e}+\mathbf{w}\|_{1}=$
0 when $t=0$. Now if $\|H t v+\mathrm{e}+\mathbf{w}\|_{1}-\|\mathrm{e}+\mathbf{w}\|_{1}>$ $0.5 \epsilon n>0$ holds true for every point $v \in Z$, we must have $\|\hat{\mathbf{x}}-\mathbf{x}\|_{2}<t$. This is because $\hat{\mathbf{x}}$ minimizes the objective in (1.3) and, $\mathbf{x}$, as a feasible solution to (1.3), gives an objective value $\|\mathrm{e}+\mathbf{w}\|_{1}$. Because we can pick arbitrarily small $t$, we must have $\|\hat{\mathbf{x}}-\mathbf{x}\|_{2} \rightarrow 0$ in probability as $n \rightarrow \infty$.

\section{Worst-Case Performance Bounds: With Out- liers Only}

We establish the worst-case performance bounds of recovering the system parameter vector $\mathbf{x}$ by $\ell_{1}$ minimization. By the performance bound, we mean that $\ell_{1}$ minimization perfectly recovers the system parameter $\mathbf{x}$ under a certain number of outliers, unlike the estimate $\hat{\mathbf{x}}$ derived in the previous section that is asymptotically convergent but never perfect.

Our main result in this regard is summarized as follows.

Theorem 3.1 Consider $y=H x+$ e. Let $H$ be an $n \times m$ Toeplitz Gaussian matrix as in (1.1), where $m$ is a fixed positive integer and $u_{i},-m+2 \leq i \leq n$ are $i . i . d . N(0,1)$ Gaussian random variables. Suppose that $\mathbf{y}=H \mathbf{x}+\mathrm{e}$, where e is a sparse outlier vector. Then there exists a constant $\beta>0$ (depending on $\mathrm{m}$ ) and a constant $c_{5}>0$ (depending on $m$ and $\beta$ ) such that, with probability at least $1-e^{-c_{5} n}$, the $n \times m$ Toeplitz matrix $H$ has the following property.

For every $\mathbf{x} \in R^{m}$ and every error e with its support $|K|$ satisfying $|K| \leq \beta n, \mathbf{x}$ is the unique solution to (1.3). Here the constant $0<\beta<1$ can be taken as any number such that, for some absolute constant number $\mu>0$ and some absolute constant number $\delta \in(0,1)$,

$$
\begin{gathered}
\beta \log (1 / \beta)+(1-\beta) \log \left(\frac{1}{1-\beta}\right)+m \beta\left[\log (2)+\frac{m \mu^{2}}{2}\right. \\
+\log (\Phi(\mu \sqrt{m}))]+\left(\frac{1}{2 m-1}-\beta\right)[\log (2) \\
\left.+\frac{1}{2} \mu^{2}(1-\delta)^{2}+\log (1-\Phi(\mu(1-\delta)))\right]<0
\end{gathered}
$$

where $\Phi(t)=\frac{1}{\sqrt{2 \pi}} \int_{-\infty}^{t} e^{-\frac{x^{2}}{2}} d x$ is the cumulative distribution function for the standard Gaussian random variable.

Remark: The derived correctable fraction of errors $\beta$ depends on the system dimension $m$.

We first outline the overall derivation strategy, and then go on to prove Theorem 3.1. Our derivation is based on checking the following now-well-known theorem for $\ell_{1}$ minimization (see [18], for example). 
Theorem 3.2 (1.3) can recover the parameter vector $\mathbf{x}$ exactly whenever $\|\mathrm{e}\|_{0} \leq k$, if and only if for every vector $z \in R^{m} \neq 0,\left\|(H z)_{K}\right\|_{1}<\left\|(H z)_{\bar{K}}\right\|_{1}$ for every subset $K \subseteq\{1,2, \ldots, n\}$ with cardinality $|K|=k$, where $\bar{K}=\{1,2, \ldots, n\} \backslash K$, and $(H z)_{K}$ is the $|K| \times 1$ vector which corresponds to the part of $\mathrm{Hz}$ over the index set $K$, and $(H z)_{\bar{K}}$ is the $(n-|K|) \times 1$ vector which corresponds to the part of $H z$ over the index set $\bar{K}$.

Similar to the average-case analysis, we adopt the strategy of discretizing the subspace generated by $H$. We first establish the property in Theorem 3.2 for all the points in $\gamma$-net $V$, before extending the property in Theorem 3.2 to $\mathrm{Hz}$ with $\|z\|_{2}=1$ not necessarily belonging to the $\gamma$-net. Following this method, we divide the derivation into Lemmas 3.3 and 3.4, which lead to Theorem 3.1. We first have Lemma 3.3 (see appendix for its proof).

Lemma 3.3 Let $\|z\|_{2}=1$ and $0<\delta<1$ be a constant. Then there exists a threshold $\beta \in(0,1)$ and a constant $c_{6}>0$ (depending on $m$ and $\beta$ ), such that with probability at least $1-e^{-c_{6} n}$, for all subsets $K \subseteq\{1,2, \ldots, n\}$ with cardinality $\frac{|K|}{n} \leq \beta$,

$$
\left\|(H z)_{K}\right\|_{1} \leq \frac{1-\delta}{2-\delta}\|H z\|_{1} .
$$

We have so far only considered the condition in Theorem 3.2 for a single point $z$ on the $\gamma$-net. By a union bound on the size of $\gamma$-net, Lemmas 7.1 and 3.3 indicate that, with overwhelming probability, the recovery condition in Theorem 3.2 holds for the discrete points on $\gamma$-net. The following lemma formally proves this fact, and then extends the proof of the recovery condition for every point in the set $\left\{z \mid\|z\|_{2}=1\right\}$.

Lemma 3.4 There exists a constant $c_{7}>0$ such that with probability at least $1-e^{-c_{7} n}$, the Gaussian Toeplitz matrix $H$ has the following property: for every $z \in R^{m}$ and every subset $K \subseteq\{1, \ldots, n\}$ with $|K| \leq \beta n$, $\sum_{i \in \bar{K}}\left|(H z)_{i}\right|-\sum_{i \in K}\left|(H z)_{i}\right| \geq \delta^{\prime} S$, where $\delta^{\prime}>0$ is a constant.

In the interest of space, we omit the detailed proof of Lemma 3.4, which is quite similar to the proof of Lemma 2.2 .

Proof (of Theorem 3.1). A direct consequence of Theorem 3.2, Lemma 3.3(together with its proof), and Lemma 3.4.

\section{Extensions to Non-Gaussian Inputs}

In previous sections, we have considered Gaussian inputs for system identifications. In fact, our results also extend

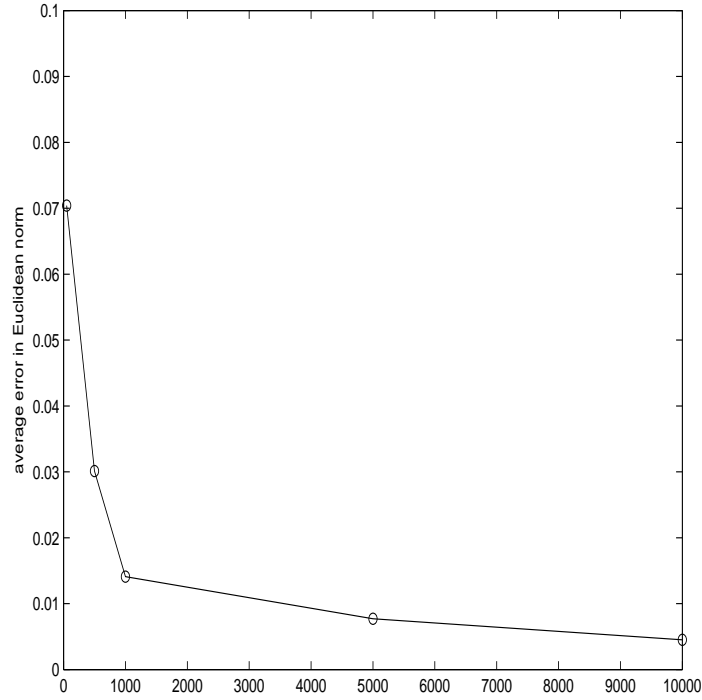

Fig. 1. Estimation errors

to non-Gaussian inputs. We illustrate this by considering a widely applied input for system identification, the pseudo-random binary sequence (PRBS) which is a random binary sequence taking values \pm 1 with the equal probability. To simplify our analysis, we only consider strong performance bounds. By noting that the PRBS input is actually an i.i.d. Bernoulli sequence, we have the following results.

Theorem 4.1 Let $H$ be an $n \times m$ Toeplitz matrix as in (1.1), where $m$ is a fixed positive integer and $u_{i},-m+$ $2 \leq i \leq n$ are i.i.d. Bernoulli random variables taking values +1 and -1 with equal probability. Suppose that $\mathbf{y}=H \mathbf{x}+\mathrm{e}$, where $\mathrm{e}$ is a sparse outlier vector with no more than $|K|=k$ non-zero elements. Then there exists a constant $c_{8}>0$ and a constant $\beta>0$ such that with probability at least $1-e^{-c_{8} n}$, the $n \times m$ Toeplitz matrix $H$ has the following property: for every $\mathbf{x} \in R^{m}$ and every error e with its support $K$ satisfying $|K|=k \leq \beta n, \mathbf{x}$ is the unique solution to (1.3).

As in Section 3, the results can be similarly shown by discretizing the subspace generated by $H$ and replacing the concentration of measure inequality for Gaussian random variables with McDiarmid's inequality [6] for binary inputs.

\section{Numerical Evaluations}

We consider a five dimensional FIR system. The input $u_{i}$ 's are an i.i.d. Gaussian sequence of zero mean and variance $2^{2}$ and the random noises are i.i.d. Gaussian of zero mean and variance $0.2^{2}$. The system parameter vector $\mathbf{x}$ is randomly generated according to Gaussian of zero mean and unit variance. The number of non-zero elements in the outlier vector is rand $\times 0.2 \times n$ where rand is the random variable uniformly in $[0,1]$ which loosely 
translates into that $10 \%$ of the data are outliers on average. The non-zero elements of the outliers are randomly generated according to Gaussian of mean $=100$ and variance $50^{2}$. The locations of the non-zeros elements are randomly assigned in each simulation. Figure 1 shows the simulation results of the estimation error for different $n$ which are the average of 10 Monte Carlo runs respectively. The numerical results support the theoretical analysis.

\section{Concluding Remarks}

We have re-visited the least absolute deviation estimator or $\ell_{1}$ minimization from a compressed sensing point of view and shown that the exact system parameter vector can be recovered as long as the outlier is sparse in the sense that the number of non-zero elements has to be bound by $\beta n$ for some $\beta<1$. No any probabilistic assumption is imposed on the unknown outlier. Further, in the presence of both the outliers and random noises, the system parameter vector can still be recovered if some mild conditions on the random noises are met.

\section{Appendix}

\subsection{Proof of Lemma 2.2}

Proof We will need Lemma 7.1, whose proof is provided in Subsection 7.2 of this appendix.

Lemma 7.1 Let $\|z\|_{2}=1$. For any $\epsilon>0$, when $n$ is large enough, with probability at least $1-2 e^{-\frac{n^{2} E^{2}\{|X|\} \epsilon^{2}}{2(n+m-1) m}}$, it holds that

$$
(1-\epsilon) S \leq\|H z\|_{1} \leq(1+\epsilon) S
$$

where $S=E\left\{\|H z\|_{1}\right\}=n E\{|X|\}$ and $X$ is a random variable following the Gaussian distribution $N(0,1)$. Namely there exists a constant $c_{9}>0$,

$$
(1-\epsilon) S \leq\|H z\|_{1} \leq(1+\epsilon) S
$$

holds with probability at least $1-2 e^{-c_{9} n}$.

For any given $\gamma>0$, there exists a $\gamma$-net $V=\left\{v_{1}, \ldots, v_{N}\right\}$ of cardinality less than $\left(1+\frac{2}{\gamma}\right)^{m}[16]$. Since each row of $H$ has $m$ i.i.d $N(0,1)$ entries, elements of $H v_{j}, 1 \leq j \leq$ $N$, are (not independent) $N(0,1)$ entries. Applying a union bound on the size of $\gamma$-net, Lemma 7.1 (Please see Lemma 7.1 and its proof in Subsection 7.2 of this appendix) implies that, for any constant $\epsilon>0$, with probability $1-2 e^{-c n}$ for some $c>0$,

$$
(1-\epsilon) S \leq\left\|H v_{j}\right\|_{1} \leq(1+\epsilon) S
$$

holds simultaneously for every vector $v_{j}$ in $V$.
For any $z$ such that $\|z\|_{2}=1$, there exists a point $v_{0}$ (we change the subscript numbering for $V$ to index the order) in $V$ such that $\left\|z-v_{0}\right\|_{2} \triangleq \gamma_{1} \leq \gamma$. Let $z_{1}$ denote $z-v_{0}$, then $\left\|z_{1}-\gamma_{1} v_{1}\right\|_{2} \triangleq \gamma_{2} \leq \gamma_{1} \gamma \leq \gamma^{2}$ for some $v_{1}$ in $V$. Repeating this process, we have $z=\sum_{j \geq 0} \gamma_{j} v_{j}$, where $\gamma_{0}=1, \gamma_{j} \leq \gamma^{j}$ and $v_{j} \in V$.

Thus for any $z \in R^{m}, z=\|z\|_{2} \sum_{j \geq 0} \gamma_{j} v_{j}$,

$$
\begin{aligned}
\sum_{i}\left|(H z)_{i}\right| & =\|z\|_{2} \sum_{i}\left|\left(\sum_{j \geq 0} \gamma_{j} H v_{j}\right)_{i}\right| \\
& \geq\|z\|_{2} \sum_{i}\left(\left|\left(H v_{0}\right)_{i}\right|-\sum_{j \geq 1} \gamma_{j}\left|\left(H v_{j}\right)_{i}\right|\right) \\
& \geq S\|z\|_{2}\left(1-\epsilon-\frac{\gamma(1+\epsilon)}{1-\gamma}\right) \\
\sum_{i}\left|(H z)_{i}\right| & \leq S\|z\|_{2}\left(1+\epsilon+\frac{\gamma(1+\epsilon)}{1-\gamma}\right)
\end{aligned}
$$

For a given $\delta$, we can pick constants $\gamma$ and $\epsilon$ small enough such that the conclusions in Lemma 2.2 hold.

\subsection{Proof of Lemma 7.1}

Proof : Our derivations rely on the concentration of measure inequalities and the Chernoff bounds for Gaussian random variables $[16,17]$.

Proposition 7.2 (Gaussian concentration inequality for Lipschitz functions) Let $f: R^{d} \rightarrow R$ be a function which is Lipschitz with constant $L$ (i.e. for all $a \in R^{d}$ and $\left.b \in R^{d},|f(a)-f(b)| \leq L\|a-b\|_{2}\right)$. Then for any $t$, we have

$$
P(|f(X)-E\{f(X)\}| \geq t) \leq 2 e^{-\frac{t^{2}}{2 L^{2}}},
$$

where $X$ is a vector of d i.i.d. standard Gaussian random variables $N(0,1)$.

We show that, for any $\|z\|_{2}=1$, the function $f(h)=\|H(h) z\|_{1}$ is a function of Lipschitz constant $\sqrt{m(n+m-1)}$, where $h=\left(u_{-m+2}, u_{-m+1}, \ldots, u_{n}\right)$, and $H(h)$ is the Toeplitz matrix in (1.1) generated from 
$h$. For two vectors $h^{1}$ and $h^{2}$, we have

$$
\begin{aligned}
\left|f\left(h^{1}\right)-f\left(h^{2}\right)\right| & =\left|\sum_{j=1}^{n}\right|\left(H\left(h^{1}\right) z\right)_{j}\left|-\sum_{j=1}^{n}\right|\left(H\left(h^{2}\right) z\right)_{j}|| \\
& \leq \sum_{i=-m+2}^{n}\left|\left(h^{1}\right)_{i}-\left(h^{2}\right)_{i}\right| \times\|z\|_{1} \\
& \leq \sqrt{n+m-1}\left\|h^{1}-h^{2}\right\|_{2} \sqrt{m}\left\|_{z}\right\|_{2} \\
& =\sqrt{(n+m-1) m}\left\|h^{1}-h^{2}\right\|_{2}
\end{aligned}
$$

by the triangular inequality. (7.1) is from the triangular inequality to $\left|\left(\left(H\left(h^{1}\right)-H\left(h^{2}\right)\right) z\right)_{j}\right|$ and the fact that $\left(h^{1}\right)_{i}-\left(h^{2}\right)_{i}$ appears in at most $m$ products with elements of $z$ (here we exploit the Toeplitz structure o $H) ;(7.2)$ is from the Cauchy-Schwarz inequality (applied twice); and (7.3) is from the fact that $\|z\|_{2}=1$. Since $\|z\|_{2}=1$, each element of $H z$ follows the standard Gaussian distribution $N(0,1)$, and $E\left\{\|H z\|_{1}\right\}=$ $n E\{|X|\}$, where $X$ follows the standard Gaussian distribution $N(0,1)$.

Then a direct application of the Gaussian concentration inequality above leads us to Lemma 7.1.

\subsection{Proof of Lemma 3.3}

Proof : We first show the concentration of measure phenomenon for $H z$, where $z \in R^{m}$ is a single vector with $\|z\|_{2}=1$.

Note that for a vector $z$ from the unit Euclidean norm in $R^{m}$, we have $\sum_{i \in K}\left|(H z)_{i}\right| \leq \sum_{i \in K} \sum_{j=1}^{m}\left|H_{i, j}\right|\left|z_{j}\right|$, and by the Cauchy-Schwarz inequality,

$$
\sum_{i \in K} \sum_{j=1}^{m}\left|H_{i, j}\right|\left|z_{j}\right| \leq \sum_{j \in J} \sqrt{m}\left|u_{j}\right|
$$

where $u_{j}$ is an element of $h=\left(u_{-m+2}, u_{-m+1}, \ldots, u_{n}\right)$ (namely the system input sequence) and $J \subseteq\{-m+$ $2, \ldots, n\}$ is the set of indices $j$ such that $u_{j}$ is involved in $H_{K}$. So the cardinality $|J| \leq m k$. By the definition of Toeplitz matrices, the number of rows in $H$ that involve only $u_{j}$ 's with $j$ coming from $\{-m+2, \ldots, n\} \backslash J$, is at least $n-k \times(2 m-1)$. We remark that the factor $(2 m-1)$ comes from the fact that each row of $H$ shares one common input with at most $(2 m-1)$ other rows. Among these rows involving only $u_{j}$ 's with $j$ coming from $\{-m+2, \ldots, n\} \backslash J$, one can pick at least $\frac{n-k \times(2 m-1)}{2 m-1}$ rows, such that these rows involve distinct $u_{j}$ 's from each other.

Thus for a fixed vector $z$, there exists a set $I \subseteq$ $\{1,2, \ldots, n\} \backslash K$ with cardinality at least $\frac{n-k \times(2 m-1)}{2 m-1}$ such that $(H z)_{i}, i \in I$, are independent $N(0,1)$ Gaussian variables; moreover, these $(H z)_{i}, i \in I$, are independent from those $u_{j}$ 's with $j \in J$. Note that for a fixed set $K$, the probability that $\left\|(H z)_{K}\right\|_{1}>\frac{1-\delta}{2-\delta}\|H z\|_{1}$ is equal to the probability that $\left\|(H z)_{K}\right\|_{1}>(1-\delta)\left\|(H z)_{K}\right\|_{1}$. Because $(1-\delta)\left\|(H z)_{\bar{K}}\right\|_{1} \geq(1-\delta)\left\|(H z)_{I}\right\|_{1}$, the probability that $\left\|(H z)_{K}\right\|_{1}>\frac{1-\delta}{2-\delta}\|H z\|_{1}$ is then no bigger than the probability $I P$ that

$$
\sqrt{m} \sum_{j \in J}\left|u_{j}\right| \geq(1-\delta) \sum_{i \in I}\left|(H z)_{i}\right| .
$$

where $u_{j}$ 's are i.i.d. $N(0,1)$ Gaussian random variables. Because $(H z)_{i}$ 's, $i \in I$, are also i.i.d. $N(0,1)$ Gaussian random variables, and moreover, they are independent of $u_{j}$ 's, $j \in J$ with $|J| \leq m k$, the probability $I P$ is no bigger than the probability

$$
\sqrt{m} \sum_{j=1}^{m k}\left|w_{j}\right| \geq(1-\delta) \sum_{i=1}^{\frac{n-k \times(2 m-1)}{2 m-1}}\left|w_{i}\right|,
$$

where $w_{i}$ 's and $w_{j}$ 's are all i.i.d. $N(0,1)$ Gaussian random variables.

Now we use the Chernoff bound [3],

$$
\begin{aligned}
& P\left(\sqrt{m} \sum_{j=1}^{m k}\left|w_{j}\right| \geq(1-\delta) \sum_{i=1}^{\frac{n-k \times(2 m-1)}{2 m-1}}\left|w_{i}\right|\right) \\
& \left.\leq \min _{\mu \geq 0} E\left\{e^{\mu\left(\sqrt{m} \sum_{j=1}^{m k}\left|w_{j}\right|-(1-\delta)\right.} \sum_{i=1}^{\frac{n-k \times(2 m-1)}{2 m-1}}\left|w_{i}\right|\right)\right\} \\
& =\min _{\mu \geq 0} E\left\{e^{\mu\left(\sqrt{m} \sum_{j=1}^{m k}\left|w_{j}\right|\right)}\right\} E\left\{e^{-\mu(1-\delta) \sum_{i=1}^{\frac{n}{2 m-1}-k}\left|w_{i}\right|}\right\}
\end{aligned}
$$

After simple algebra, we have

$$
E\left\{e^{\mu\left(\sqrt{m}\left|w_{j}\right|\right)}\right\}=2 e^{\frac{\mu^{2} m}{2}} \Phi(\mu \sqrt{m})
$$

and

$$
E\left\{e^{-\mu(1-\delta)\left|w_{i}\right|}\right\}=2 e^{\frac{\mu^{2}(1-\delta)^{2}}{2}}(1-\Phi(\mu(1-\delta)))
$$

where $\Phi(t)$ is the cumulative distribution function for a standard Gaussian random variable $N(0,1)$.

Putting this back into the Chernoff bound and noticing that there are at most $\left(\begin{array}{l}n \\ k\end{array}\right)$ possible support sets $K$ with 
cardinality $k$, the logarithm of the probability $P$ that $\sqrt{m} \sum_{j=1}^{m k}\left|w_{j}\right|<(1-\delta) \sum_{i=1}^{\frac{n-k \times(2 m-1)}{2 m-1}}\left|w_{i}\right|$ is violated for at least one support set $K$ is upper bounded, by the union bound,

$$
\begin{aligned}
& \log \left(\left(\begin{array}{l}
n \\
k
\end{array}\right)\right)+m k\left[\log (2)+\frac{m \mu^{2}}{2}+\log (\Phi(\mu \sqrt{m}))\right] \\
& +\left(\frac{n}{2 m-1}-k\right)\left[\log (2)+\frac{\mu^{2}(1-\delta)^{2}}{2}+\log (Q((1-\delta) \mu))\right],
\end{aligned}
$$

where $Q((1-\delta) \mu)=1-\Phi((1-\delta) \mu)$ is the Gaussian tail function.

Let $k=\beta n$, and thus $\log \left(\left(\begin{array}{l}n \\ k\end{array}\right)\right) / n \rightarrow H(\beta)$ as $n \rightarrow \infty$, where $H(\beta)=\beta \log (1 / \beta)+(1-\beta) \log \left(\frac{1}{1-\beta}\right)$ is the entropy function. As long as, for a certain $\mu>0$,

$$
\begin{aligned}
& H(\beta)+m \beta \times\left[\log (2)+\frac{m \mu^{2}}{2}+\log (\Phi(\mu \sqrt{m}))\right]+ \\
& \left(\frac{1}{2 m-1}-\beta\right)\left[\log (2)+\frac{\mu^{2}(1-\delta)^{2}}{2}+\log (Q(\mu(1-\delta)))\right] \\
& <0,
\end{aligned}
$$

then $\beta$ is within the correctable error region for all the support sets $K$ with high probability. In fact, the last quantity can always be made smaller than 0 if we take $\beta$ small enough. To see this, we can first take $\mu$ large enough, such that $\left[\log (2)+\frac{1}{2} \mu^{2}(1-\delta)^{2}+\log (1-\Phi(\mu(1-\right.$ $\delta)))]<0$. This is always doable, because the tail function $1-\Phi(\mu(1-\delta))$ satisfies

$$
1-\Phi(\mu(1-\delta)) \leq \frac{\frac{1}{\sqrt{2 \pi}} e^{-\frac{\mu^{2}(1-\delta)^{2}}{2}}}{\mu(1-\delta)}
$$

for $\mu(1-\delta)>0$. Fixing that $\mu$, we can then take $\beta$ sufficiently small such that $\log (P)<0$, as $n \rightarrow \infty$.

\section{References}

[1] E.W. Bai, H. Cho, R. Tempo and Y. Ye, "Optimization with a few violated constraints and its application in bounded error system identification", IEEE Trans on Automatic Control, Vol. 42, pp.1067-1077, 2002.

[2] X. Chen, Z. Bai and L. Zao, Asymptotic normality of minimum $L_{1}$ Norm Estimates in Linear Models, Science of China, Vol. 33, No. 11, pp.1311-1328, 1990.

[3] H. Chernoff, A Measure of Asymptotic Efficiency for Tests of a Hypothesis Based on the sum of Observations, Annals of Mathematical Statistics, vol. 23(4): 493-507,1952.

[4] P. Rousseeuw and A. Leroy, Robust Regression and Outlier Detection, John Wiley, NY,1987.

[5] L. Ljung, System Identification: Theory for the User, Prentice-Hall, Englewood Cliffs, NJ,1987.
[6] C. McDiarmid, "On the Method of Bounded Differences," Surveys in Combinatorics vol. 141, pp.148-188,1989.

[7] T. Söderström and P. Stoica, System Identification, PrenticeHall, Englewood Cliffs, NJ,1989.

[8] E. Candès and P. Randall, "Highly robust error correction by convex programming," IEEE Transactions on Information Theory, vol. 54, pp. 2829-2840, 2008.

[9] E. Candès and T. Tao, "Decoding by linear programming", IEEE Trans. on Information Theory, 51(12), pp. 4203 - 4215, December 2005.

[10] D. Donoho, "High-dimensional centrally symmetric polytopes with neighborliness proportional to dimension," Discrete and Comput. Geom., vol. 35, no. 4, pp. 617-652, 2006.

[11] D. Donoho and J. Tanner, "Neighborliness of randomly projected simplices in high dimensions," in Proc. Natl. Acad. Sci. USA, vol. 102, no. 27, pp. 9452-9457,2005.

[12] P. Huber, Robust Statistics, Wiley, New York, 1981

[13] M. Stojnic, W. Xu, and B. Hassibi, "Compressed sensing probabilistic analysis of a null space characterization," in Proc. Int. Conf. Acoustics, Speech, Signal Processing (ICASSP), 2008.

[14] J. Romberg, "Compressed sensing by random convolution," SIAM Journal on Imaging Sciences, vol. 2, no. 4, pp. 1098 1128, 2009.

[15] H. Rauhut, "Circulant and Toeplitz matrices in compressed sensing," in Proc. SPARS'09, Saint-Malo, France, 2009.

[16] M. Ledoux, The Concentration of Measure Phenomenon, American Mathematical Society,2001.

[17] M. Ledoux and M. Talagrand, Probability in Banach Spaces, Berlin: Springer-Verlag, 1991

[18] W. Xu and B. Hassibi, "Precise Stability Phase Transitions for $\ell_{1}$ Minimization: A Unified Geometric Framework," IEEE Transactions on Information Theory, vol. 57, pp. 6894-6919, 2011.

[19] W. Xu, M. Wang, and A. Tang, "On state estimation with bad data detection," Proceedings of IEEE Conference on Decision and Control, 2011.

[20] Y. Chen, Y. Gu and A. O. Hero, "Sparse LMS for system identification," Proceedings of IEEE ICASSP, pp. 3125-3128, Taipei, Taiwan, 2009.

[21] Y. Kopsinis, K. Slavakis, and S. Theodoridis, "Online sparse system identification and signal reconstruction using projections onto weighted $\ell_{1}$ balls," IEEE Trans. on Signal Processing, pp.936-952, Mar. 2011

[22] B. Sanandaji, T. Vincent, K. Poolla, and M. Wakin, "A tutorial on recovery conditions for compressive system identification of sparse channels," IEEE 51st Annual Conference on Decision and Control (CDC), 2012. 